\title{
Insider Trading in Convergent Markets
}

\author{
Mattias Jonsson, University of Michigan, Department of Mathematics, Ann Arbor, MI 48109- \\ 1109 (mattiasj@umich.edu) \\ Jan Večeř, Columbia University, Department of Statistics, New York, NY 10027 \\ (vecer@stat.columbia.edu)
}

September 16, 2003

\begin{abstract}
We find optimal trading strategies for an insider who is trading in two convergent stocks and is bound by margin constraints.
\end{abstract}

\section{Introduction}

The purpose of this paper is to find optimal strategies for trading in the stocks of two companies (or the currencies of two countries) which will merge in the future (or join a monetary union in the case of currencies). When the merger happens at time $T$, we assume that the ratio of the two stock prices will be equal to some pre-specified constant $C>0$ :

$$
\frac{S_{T}^{1}}{S_{T}^{2}}=C
$$

The constant $C$ is the ratio in which the two companies exchange their old stocks for the newly created merged company. We assume that this information is available to the insider, but not to the rest of the market. There are many examples of convergent markets when there are two or more processes (stock prices, exchange rates or interest rates) for which one can have some information about their relative future evolution in the above form.

If otherwise unrestricted, the insider may achieve unbounded wealth in finite time if he or she has enough additional information in comparison to the rest of the market. This is clearly something that we wish to rule out. One possible recent approach presented in Hu and Øksendal [HO is to penalize trading strategies which are not smooth. In our paper, we follow the approach of Liu and Longstaff $[\mathrm{LL}$, and put constraints on the margin account positions. We assume that the only additional information available to the insider is that the merger will happen at time $T$ as described in equation (1). We use a model where the two stocks are driven by two possibly correlated Brownian motions and we consider strategies that maximize expected terminal wealth or expected logarithm of terminal wealth. The strategies are restricted by margin constraints that bound the short positions in terms of the current wealth. Interestingly enough, the optimal strategy is often different from simply locking the arbitrage opportunity. See Theorems 2.1, 2.3 and 2.4.

Insider trading has previously been studied from many different points of view. Generally speaking, the insider is assumed to have a larger information set (filtration) $\mathbb{H}=\left\{\mathcal{H}_{t}\right\}_{0 \leq t \leq T}$ than the information $\mathbb{G}=\left\{\mathcal{G}_{t}\right\}_{0 \leq t \leq T}$ available to the rest of the market. Karatzas and Pikovski $[\mathrm{KP}$ studied a problem of optimal investment for strategies available to the insider ( $\mathcal{H}_{t}$-adapted) for 
a logarithmic choice of utility function. They use the result from the enlargement of filtration of Jeulin and Yor [JY]. Subsequent work includes papers of Leon, Navarro and Nualart [LNN], or Imkeller [ []. Recently, a general stochastic approach to this problem is presented in Biagini and Øksendal [BO].

While many of the papers just mentioned work in a quite general setup, they focus on markets with a single risky instrument. Our setup, on the other hand, naturally takes into account the common geometric Brownian motion assumption on the two price processes together with the merger information encapsulated in (1). We also obtain explicit expressions for the optimal trading strategies.

As we show, the merger is equivalent to the conditioning on the final position of the linear combination of the two Brownian motions governing the stock price processes. This is very similar to the Brownian bridge process, except that the process is now two-dimensional. We refer to it as a planar Brownian bridge process and we express it as the solution to an SDE driven by two independent Brownian motions. This leads to an SDE for the two stock prices, and thus to the dynamics of wealth, from which we find the optimal strategies. An independent (and different) application of two (conditioned) Brownian motions to insider trading was studied by Föllmer, Wu and Yor [FWY].

\section{Results}

In this section we give the precise setup for our analysis and present the main results of the paper giving optimal strategies for trading in convergent markets. The proofs will be given in Section 3 .

\subsection{Setup and main results}

Consider an investor trading in two stocks of companies that are about to merge, as well as in a bank account. The stock prices evolve according to geometric Brownian motion

$$
\begin{aligned}
\frac{d S_{t}^{1}}{S_{t}^{1}} & =\mu_{1} d t+\sigma_{1} d W_{t}^{1} \\
\frac{d S_{t}^{2}}{S_{t}^{2}} & =\mu_{2} d t+\sigma_{2} d W_{t}^{2}
\end{aligned}
$$

where $W_{t}^{1}$ and $W_{t}^{2}$ are two correlated Brownian motions with $d W_{t}^{1} \cdot d W_{t}^{2}=\rho d t,-1<\rho<1$, and where we condition on the merger event

$$
S_{T}^{1}=C \cdot S_{T}^{2}
$$

We assume that the interest rate is zero, something that can be achieved by appropriate discounting. The initial wealth $Y_{0}$ of the investor is fixed and the wealth $Y_{t}^{\pi}$ then follows

$$
\frac{d Y_{t}^{\pi}}{Y_{t}^{\pi}}=\pi_{t}^{1} \frac{d S_{t}^{1}}{S_{t}^{1}}+\pi_{t}^{2} \frac{d S_{t}^{2}}{S_{t}^{2}}
$$

where $\pi_{t}^{i}$ is the fraction of wealth invested in stock $i$. Thus $1-\pi_{t}^{1}-\pi_{t}^{2}$ is the fraction of wealth held in the money market account. See [KS2].

The objective of the investor is to maximize his expected utility of wealth subject to some trading constraints.

We first consider the case where no borrowing or short selling is allowed. In other words, $\pi_{t}^{1}, \pi_{t}^{2} \geq 0$ and $\pi_{t}^{1}+\pi_{t}^{2} \leq 1$. The total wealth $Y_{t}$ then automatically remains non-negative. Let us first assume that the investor wants to maximize the expected wealth, i.e. using he/she has a linear utility function. 
Theorem 2.1 The strategy $\pi_{t}$ maximizing $E\left[Y_{T}^{\pi}\right]$ using no borrowing or short-selling always satisfies $\pi_{t} \in\{(0,0),(1,0),(0,1)\}$ and only depends on $X_{t}:=\frac{1}{T-t} \log \frac{S_{t}^{1}}{C S_{t}^{2}}$. More precisely, define the planar process $Z_{t}:=\left(Z_{t}^{1}, Z_{t}^{2}\right):=\left(-A_{1} X_{t}+B_{1}, A_{2} X_{t}+B_{2}\right)$, where

$$
\begin{gathered}
A_{1}=\frac{\sigma_{1}\left(\sigma_{1}-\rho \sigma_{2}\right)}{\sigma_{1}^{2}-2 \rho \sigma_{1} \sigma_{2}+\sigma_{2}^{2}} \quad \text { and } \quad A_{2}=\frac{\sigma_{2}\left(\sigma_{2}-\rho \sigma_{1}\right)}{\sigma_{1}^{2}-2 \rho \sigma_{1} \sigma_{2}+\sigma_{2}^{2}} \\
B_{1}=\mu_{1} A_{2}+\left(\mu_{2}+\frac{1}{2}\left(\sigma_{1}^{2}-\sigma_{2}^{2}\right)\right) A_{1} \quad \text { and } \quad B_{2}=\mu_{2} A_{1}+\left(\mu_{1}+\frac{1}{2}\left(\sigma_{2}^{2}-\sigma_{1}^{2}\right)\right) A_{2} .
\end{gathered}
$$

Then

$$
\pi_{t}=\left\{\begin{array}{l}
(0,0) \text { if } Z_{t}^{1} \leq 0 \text { and } Z_{t}^{2} \leq 0 \\
(1,0) \text { if } Z_{t}^{1} \geq 0 \text { and } Z_{t}^{2} \leq Z_{t}^{1} \\
(0,1) \text { if } Z_{t}^{2} \geq 0 \text { and } Z_{t}^{1} \leq Z_{t}^{2}
\end{array}\right.
$$

Remark 2.2 The optimal strategy is depicted in Figure 2. The apparent inconsistency in the definition (6) of the optimal strategy is to be interpreted as follows: in the (probability zero) cases when the definition above is inconsistent, then the optimal strategy is not unique. For instance, if $Z_{t}=(1,1)$, then $\pi_{t}=(1,0)$ and $\pi_{t}=(0,1)$ are both optimal strategies.

Theorem 2.1 can be generalized to trading constraints of the form

$$
\text { (total short positions) } \leq \lambda \text { (total wealth) }
$$

where $\lambda \geq 0$ is a fixed number. This is a margin constraint which can be written as $\pi_{t} \in \Omega_{\lambda}$ where $\Omega_{\lambda}$ is the convex set illustrated in Figure 1 . The earlier constraint corresponds to $\lambda=0$.



Figure 1: The margin constraint restricts the strategy $\left(\pi_{t}^{1}, \pi_{t}^{2}\right)$ to be in the convex set $\Omega_{\lambda}$ bounded by the dotted lines.

Theorem 2.3 The strategy $\pi_{t}$ maximizing $E\left[Y_{T}^{\pi}\right]$ subject to the margin constraint (7) only depends on $X_{t}:=\frac{1}{T-t} \log \frac{S_{t}^{1}}{C S_{t}^{2}}$ and satisfies $\pi_{t} \in\{(-\lambda, 0),(0,-\lambda),(1+\lambda,-\lambda),(-\lambda, 1+\lambda),(1+\lambda, 0),(0,-\lambda)\}$. More precisely, if $Z_{t}=\left(Z_{t}^{1}, Z_{t}^{2}\right)$ is as in Theorem 2.1 then $\pi_{t}$ is given by Figure 2

The precise behavior of the optimal strategies in Theorems 2.1 and 2.3 as functions of $X_{t}$ (or $S_{t}$ ) depend on the constants $A_{i}$ and $B_{i}$. Rather than going through all possibilities, we illustrate one particular scenario in Figure 3 .

Finally we turn to the case of a logarithmic utility function. 



Figure 2: The strategies maximizing expected wealth, illustrating Theorems 2.1 (left) and 2.3 (right). The vector $Z_{t}$ is given in Theorem 2.1 and depends only on $\frac{1}{T-t} \log \frac{S_{t}^{1}}{C S_{t}^{2}}$. For instance, if $Z_{t}$ belongs to the second quadrant, then $\pi_{t}=(-\lambda, 1+\lambda)$ in Theorem 2.3 and $\pi_{t}=(0,1)$ in Theorem 2.1 (which corresponds to $\lambda=0$ ). In other words, the optimal strategy is to go short a fraction $\lambda$ of the current wealth in the first stock, go long a fraction $1+\lambda$ of the current wealth in the second stock and put no money in the bank account.

Theorem 2.4 There is a unique strategy $\pi_{t}^{*}$ maximizing $E\left[\log Y_{T}^{\pi}\right]$ subject to the margin constraint (7). The strategy $\pi_{t}^{*}$ only depends on $X_{t}:=\frac{1}{T-t} \log \frac{S_{t}^{1}}{C S_{t}^{2}}$. Moreover, $\pi_{t}^{*}$ is a continuous and piecewise affine function of $X_{t}$.

The exact form of the optimal strategy $\pi_{t}$ in Theorem 2.4 as a function of $X_{t}$ (or of $S_{t}$ ) depends on the constants $A_{i}$ and $B_{i}$. We illustrate one particular case in Figure 4

\subsection{Special case: one convergent stock}

Now consider trading in a single stock with dynamics $d S_{t}=S_{t}\left(\mu d t+\sigma d W_{t}\right)$, conditioned on $S_{T}=C$. The trading strategy is $\pi_{t}$, i.e. the fraction of the wealth put into the stock. We may view this as a special case of the above, by putting $S_{t}^{1}=S_{t}, \pi_{t}^{1}=\pi_{t}, \sigma_{2}=\mu_{2}=\pi_{t}^{2}=0$ and $S_{t}^{2} \equiv 1$. The margin condition (7) becomes

$$
-\lambda \leq \pi_{t} \leq 1+\lambda
$$

Let us first consider linear utility.

Corollary 2.5 The strategy trading in one convergent stock and maximizing $E\left[Y_{T}^{\pi}\right]$ subject to the margin condition (8) is given by

$$
\pi_{t}= \begin{cases}-\lambda & \text { if } S_{t} \geq C \exp \left(\frac{1}{2} \sigma^{2}(T-t)\right) \\ 1+\lambda & \text { if } S_{t} \leq C \exp \left(\frac{1}{2} \sigma^{2}(T-t)\right)\end{cases}
$$

In the case of logarithmic utility, the result is the following.

Corollary 2.6 The strategy trading in one convergent stock and maximizing $E\left[\log Y_{T}^{\pi}\right]$ subject to the margin constraint (8) is given by

$$
\pi_{t}= \begin{cases}-\lambda & \text { if } \quad X_{t} \geq \sigma^{2}\left(\frac{1}{2}+\lambda\right) \\ \frac{1}{2}-\frac{1}{\sigma^{2}} X_{t} & \text { if } \quad-\sigma^{2}\left(\frac{1}{2}+\lambda\right) \leq X_{t} \leq \sigma^{2}\left(\frac{1}{2}+\lambda\right) \\ 1+\lambda & \text { if } \quad X_{t} \leq-\sigma^{2}\left(\frac{1}{2}+\lambda\right)\end{cases}
$$



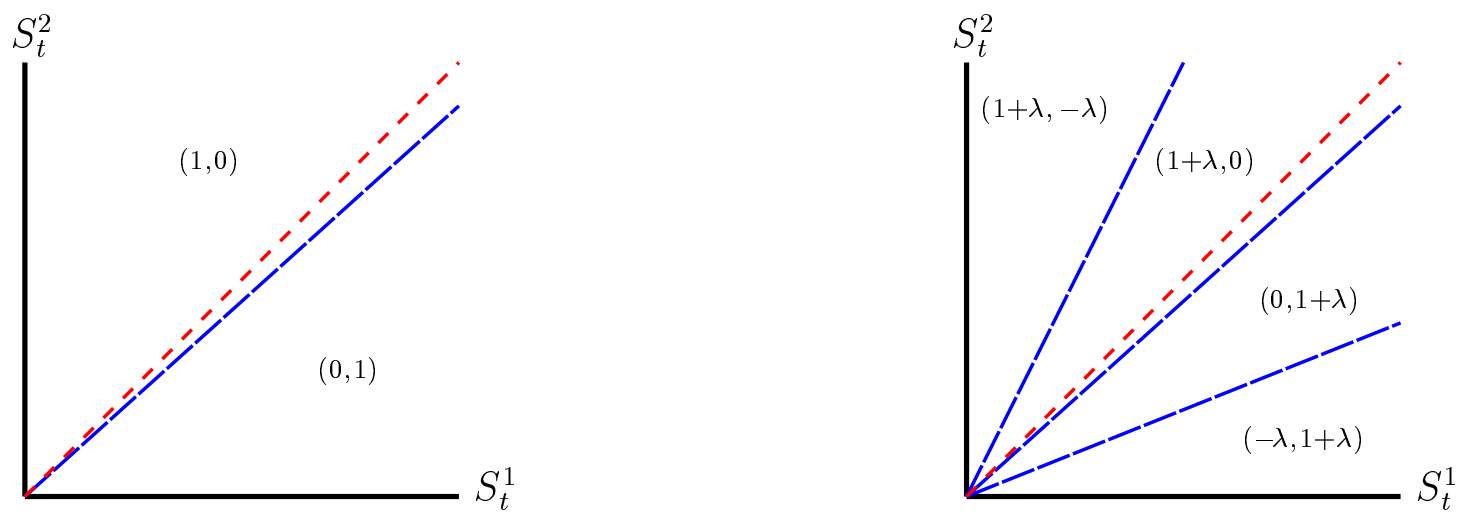

Figure 3: The strategies in Theorems 2.1 and2.3 are shown as functions of $S_{t}$, for given parameters. The dotted lines represent $S_{t}^{1}=C S_{t}^{2}$, to which all lines converge as $t \rightarrow T$. Notice that it is possible that $S_{t}^{1}>C S_{t}^{2}$ and that the optimal strategy is to go long $S_{1}$ and hold no $S_{2}$, even though the ratio $S_{t}^{1} / S_{t}^{2}$ is bound to decrease. We used $\sigma_{1}=0.3, \sigma_{2}=0.3, \rho=0.2, \mu_{1}=0.3, \mu_{2}=0.2, C=1$ and $T-t=0.5$ to get these pictures (qualitatively).

where $X_{t}=\frac{1}{T-t} \log \frac{S_{t}}{C}$.

Remark 2.7 As in Remark 2.2 the optimal strategies in Corollaries 2.5 and 2.6 are somewhat surprising. In particular it can happen that the optimal strategy consists of holding the stock even while knowing that the stock price eventually will drop.

\section{Proofs}

We now turn to the proofs of the main results. The idea is that the conditioning (1) leads to a new SDE for the two stock price processes.

\subsection{The one-dimensional Brownian bridge}

Conditioning a Brownian motion $\left(X_{t}\right)_{0 \leq t \leq T}$ on its terminal value $X_{T}$ leads to a Brownian bridge [KS1]: if the starting value is $X_{0}=a$ and the end value is $X_{T}=b$, then

$$
d X_{t}=\frac{b-X_{t}}{T-t} d t+d W_{t}, \quad X_{0}=a .
$$

\subsection{The planar Brownian bridge}

We now derive the dynamics of a planar Brownian bridge, i.e. the two-dimensional version of (9).

Proposition 3.1 Suppose that $W_{t}^{1}, W_{t}^{2}$ are two Brownian motions starting at $W_{0}^{1}=W_{0}^{2}=0$, with instantaneous correlation $d W_{t}^{1} \cdot d W_{t}^{2}=\rho d t$, and conditioned on

$$
a_{1} W_{T}^{1}+a_{2} W_{T}^{2}=b
$$




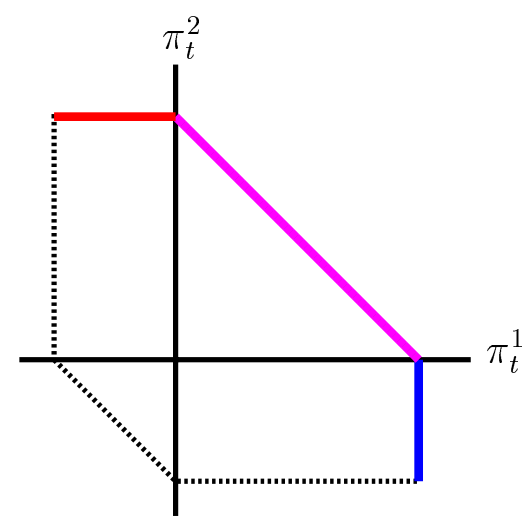

Figure 4: The strategy maximizing expected logarithm of wealth with the margin constraint (7). We used constants $\mu_{1}=0.1, \mu_{2}=0.15, \sigma_{1}=0.2, \sigma_{2}=0.3, \rho=0.1, C=1, t-T=0.5, \lambda=0.5$. The optimal constrained strategy (Theorem 2.4) lies on the three upper-right line segments and travels from right (down) to left (up) as $X_{t}$ increases. For $X_{t} \leq-0.2359,-0.1734 \leq X_{t} \leq-0.0751$, $0.1001 \leq X_{t} \leq 0.3412$ and $X_{t} \geq 0.4098, \pi_{t}$ is constant: $\pi_{t}=(1+\lambda,-\lambda), \pi_{t}=(1+\lambda, 0)$, $\pi_{t}=(0,1+\lambda)$ and $\pi_{t}=(-\lambda, 1+\lambda)$, respectively. For all other values of $X_{t}, \pi_{t}$ is a nonconstant affine function of $X_{t}$.

where $a_{i}$ and $b$ are constants. Than the dynamics of $W_{t}^{1}, W_{t}^{2}$ can be written as

$$
\begin{aligned}
& d W_{t}^{1}=\left(a_{1}+\rho a_{2}\right) \frac{b-a_{1} W_{t}^{1}-a_{2} W_{t}^{2}}{(T-t)\left(a_{1}^{2}+2 \rho a_{1} a_{2}+a_{2}^{2}\right)} d t+\frac{a_{1}+\rho a_{2}}{\sqrt{a_{1}^{2}+2 \rho a_{1} a_{2}+a_{2}^{2}}} d \xi_{t}^{1}-\frac{a_{2} \sqrt{1-\rho^{2}}}{\sqrt{a_{1}^{2}+2 \rho a_{1} a_{2}+a_{2}^{2}}} d \xi_{t}^{2} \\
& d W_{t}^{2}=\left(a_{2}+\rho a_{1}\right) \frac{b-a_{1} W_{t}^{1}-a_{2} W_{t}^{2}}{(T-t)\left(a_{1}^{2}+2 \rho a_{1} a_{2}+a_{2}^{2}\right)} d t+\frac{a_{2}+\rho a_{1}}{\sqrt{a_{1}^{2}+2 \rho a_{1} a_{2}+a_{2}^{2}}} d \xi_{t}^{1}+\frac{a_{1} \sqrt{1-\rho^{2}}}{\sqrt{a_{1}^{2}+2 \rho a_{1} a_{2}+a_{2}^{2}}} d \xi_{t}^{2}
\end{aligned}
$$

where $\xi_{t}^{1}$ and $\xi_{t}^{2}$ are two independent standard Brownian motions.

Proof Let us introduce two new processes:

$$
\begin{aligned}
& U_{t}^{1}=a_{1} W_{t}^{1}+a_{2} W_{t}^{2}, \\
& U_{t}^{2}=-\left(a_{2}+\rho a_{1}\right) W_{t}^{1}+\left(a_{1}+\rho a_{2}\right) W_{t}^{2} .
\end{aligned}
$$

It is easy to show that $U_{t}^{1}$ and $U_{t}^{2}$ are independent. Now $U_{t}^{1} / \sqrt{a_{1}^{2}+2 \rho a_{1} a_{2}+a_{2}^{2}}$ is the conditioning of a Brownian motion on its terminal value and is hence a Brownian bridge. Further, $U_{t}^{2} / \sqrt{\left(1-\rho^{2}\right)\left(a_{1}^{2}+2 \rho a_{1} a_{2}+a_{2}^{2}\right)}$ is a Brownian motion. Thus we can write dynamics of $U_{t}^{1}$ and $U_{t}^{2}$ in the form

$$
\begin{aligned}
& d U_{t}^{1}=\frac{b-U_{t}^{1}}{T-t} d t+\sqrt{a_{1}^{2}+2 \rho a_{1} a_{2}+a_{2}^{2}} d \xi_{t}^{1} \\
& d U_{t}^{2}=\sqrt{1-\rho^{2}} \sqrt{a_{1}^{2}+2 \rho a_{1} a_{2}+a_{2}^{2}} d \xi_{t}^{2}
\end{aligned}
$$

where $B_{t}^{1}, B_{t}^{2}$ are independent standard Brownian motions. This leads to

$$
\begin{aligned}
d\left(a_{1} W_{t}^{1}+a_{2} W_{t}^{2}\right) & =\frac{b-a_{1} W_{t}^{1}-a_{2} W_{t}^{2}}{T-t} d t+\sqrt{a_{1}^{2}+2 \rho a_{1} a_{2}+a_{2}^{2}} d \xi_{t}^{1} \\
d\left(-\left(a_{2}+\rho a_{1}\right) W_{t}^{1}+\left(a_{1}+\rho a_{2}\right) W_{t}^{2}\right) & =\sqrt{1-\rho^{2}} \sqrt{a_{1}^{2}+2 \rho a_{1} a_{2}+a_{2}^{2}} d \xi_{t}^{2} .
\end{aligned}
$$

Now we can solve for $d W_{t}^{1}$ and $d W_{t}^{2}$ to obtain (11) and (12). 


\subsection{Stock Dynamics}

Using the planar Brownian bridge we now derive the dynamics of two merging stocks.

Proposition 3.2 Assume that the dynamics of two stock prices is given by

$$
\begin{aligned}
& \frac{d S_{t}^{1}}{S_{t}^{1}}=\mu_{1} d t+\sigma_{1} d W_{t}^{1} \\
& \frac{d S_{t}^{2}}{S_{t}^{2}}=\mu_{2} d t+\sigma_{2} d W_{t}^{2}
\end{aligned}
$$

where $W_{t}^{1}$ and $W_{t}^{2}$ are two correlated Brownian motions with $d W_{t}^{1} \cdot d W_{t}^{2}=\rho d t$, and where we condition on the event

$$
S_{T}^{1}=C \cdot S_{T}^{2}
$$

Then the stock price dynamics can be expressed as

$$
\begin{aligned}
& \frac{d S_{t}^{1}}{S_{t}^{1}}=\left(-A_{1} X_{t}+B_{1}\right) d t+C_{1} d B_{t}^{1}+D d \xi_{t}^{2} \\
& \frac{d S_{t}^{1}}{S_{t}^{1}}=\left(A_{2} X_{t}+B_{2}\right) d t-C_{2} d B_{t}^{1}+D d \xi_{t}^{2}
\end{aligned}
$$

where $\xi_{t}^{1}$ and $\xi_{t}^{2}$ are two independent standard Brownian motions, where $X_{t}, A_{1}, A_{2}, B_{1}, B_{2}$ are as in Theorem 2.1 and where

$$
C_{1}=\frac{\sigma_{1}\left(\sigma_{1}-\rho \sigma_{2}\right)}{\sqrt{\sigma_{1}^{2}-2 \rho \sigma_{1} \sigma_{2}+\sigma_{2}^{2}}}, \quad C_{2}=\frac{\sigma_{2}\left(\sigma_{2}-\rho \sigma_{1}\right)}{\sqrt{\sigma_{1}^{2}-2 \rho \sigma_{1} \sigma_{2}+\sigma_{2}^{2}}} \quad \text { and } \quad D=\frac{\sigma_{1} \sigma_{2} \sqrt{1-\rho^{2}}}{\sqrt{\sigma_{1}^{2}-2 \rho \sigma_{1} \sigma_{2}+\sigma_{2}^{2}}}
$$

Proof. For geometric Brownian motion we have

$$
S_{t}^{i}=S_{0}^{i} \exp \left(\left(\mu_{i}-\frac{1}{2} \sigma_{i}^{2}\right) t+\sigma_{i} W_{t}^{i}\right) \quad i=1,2 .
$$

Therefore the condition $S_{T}^{1} / S_{T}^{2}=C$ translates as

$$
\sigma_{1} W_{T}^{1}-\sigma_{2} W_{T}^{2}=\left(\mu_{2}-\mu_{1}-\frac{1}{2}\left(\sigma_{2}^{2}-\sigma_{1}^{2}\right)\right) T+\log \left(\frac{C S_{0}^{2}}{S_{0}^{1}}\right) .
$$

This identity defines a planar Brownian bridge with

$$
a_{1}=\sigma_{1}, \quad a_{2}=-\sigma_{2} \quad \text { and } \quad b=\left(\mu_{2}-\mu_{1}-\frac{1}{2}\left(\sigma_{2}^{2}-\sigma_{1}^{2}\right)\right) T+\log \left(\frac{C S_{0}^{2}}{S_{0}^{1}}\right) .
$$

The dynamics of the planar Brownian bridge was given in Proposition 3.1 in terms of two independent standard Brownian motions $\xi_{t}^{1}$ and $\xi_{t}^{2}$. Plugging expressions $(11)$ and $(12)$ into $(13)$ and (14), we conclude the proof.

Corollary 3.3 Assume that a single stock follows the dynamics

$$
\frac{d S_{t}}{S_{t}}=\mu d t+\sigma d W_{t}
$$

conditioned on $S_{T}=C$. Then

$$
\frac{d S_{t}}{S_{t}}=\left(\frac{1}{T-t} \log \frac{S_{T}}{S_{t}}+\frac{1}{2} \sigma^{2}\right) d t+\sigma d \xi_{t},
$$

where $\xi_{t}$ is a standard Brownian motion.

Proof. Special case of Proposition 3.2 with $S_{t}=S_{t}^{1}, \mu=\mu_{1}, \sigma=\sigma_{1}, \mu_{2}=\sigma_{2}=0, S_{t}^{2} \equiv 1$.

Remark 3.4 Notice that the actual drift $\mu$ has no relevance to the dynamics of the stock price if we condition on its terminal price. 


\subsection{Optimal strategies}

We are now ready to prove the main results.

Proof of Theorem 2.1. By (15) and (16) we can write

$$
\begin{aligned}
E\left[Y_{T}^{\pi}\right] & =Y_{0}+E\left[\int_{0}^{T}\left(\pi_{t}^{1} \frac{d S_{t}^{1}}{S_{t}^{1}}+\pi_{t}^{2} \frac{d S_{t}^{2}}{S_{t}^{2}}\right) Y_{t}^{\pi}\right] \\
& =Y_{0}+E\left[\int_{0}^{T}\left(\pi_{t}^{1}\left(-A_{1} X_{t}+B_{1}\right)+\pi_{t}^{2}\left(A_{2} X_{t}+B_{2}\right)\right) Y_{t}^{\pi} d t\right] \\
& =Y_{0}+E\left[\int_{0}^{T}\left\langle\pi_{t}, Z_{t}\right\rangle Y_{t}^{\pi} d t\right]
\end{aligned}
$$

since the $d \xi$-integrals have expected value zero. Here $\langle\cdot, \cdot\rangle$ denotes the standard inner product in $\mathbf{R}^{2}$. Recall that $Y_{t}^{\pi} \geq 0$. Thus the two integrals are maximized if the quantity $\left\langle\pi_{t}, Z_{t}\right\rangle$ is maximized for each $t$, subject to the constraint that $\pi_{t}$ is an admissible strategy. It is now easy to check that this leads to the formula in Theorem 2.1, depicted in Figure 2, The proof is complete.

Proof of Theorem 2.3. The proof is exactly the same as above: we are still maximizing the quantity $\left\langle\pi_{t}, Z_{t}\right\rangle$ for each $t$, but the strategy $\pi_{t}$ is allowed to be in the larger set $\Omega_{\lambda}$ depicted in Figure 1 .

Proof of Theorem 2.4. We use Itô's formula and Proposition 3.2 to compute

$$
\begin{aligned}
d \log Y_{t}^{\pi} & =\frac{d Y_{t}^{\pi}}{Y_{t}^{\pi}}-\frac{1}{2}\left(\frac{d Y_{t}^{\pi}}{Y_{t}^{\pi}}\right)^{2} \\
& =\left(\pi_{t}^{1} \frac{d S_{t}^{1}}{S_{t}^{1}}+\pi_{t}^{2} \frac{d S_{t}^{2}}{S_{t}^{2}}\right)-\frac{1}{2}\left(\pi_{t}^{1} \frac{d S_{t}^{1}}{S_{t}^{1}}+\pi_{t}^{2} \frac{d S_{t}^{2}}{S_{t}^{2}}\right)^{2} \\
& =Q_{t}\left(\pi_{t}^{1}, \pi_{t}^{2}\right) d t+\star d B_{t}^{1}+\star d B_{t}^{2}
\end{aligned}
$$

where $\star$ are coefficients that are irrelevant for us, and where $Q_{t}$ is a quadratic function given by

$$
Q_{t}(\pi)=\pi^{1}\left(-A_{1} X_{t}+B_{1}\right)+\pi^{2}\left(A_{2} X_{t}+B_{2}\right)-\frac{1}{2}\left(C_{1} \pi^{1}-C_{2} \pi^{2}\right)^{2}-\frac{1}{2} D^{2}\left(\pi^{1}+\pi^{2}\right)^{2}
$$

Notice that $Q_{t}$ depends only on $X_{t}$ (and on constants). Also notice that the pure quadratic part of $Q_{t}$ is strictly negative definite, since if $\pi^{1}+\pi^{2}=0$ and $C_{1} \pi^{1}-C_{2} \pi^{2}=0$, then $\left(C_{1}+C_{2}\right) \pi_{1}=0$. But $C_{1}+C_{2}=\sqrt{\sigma_{1}^{2}-2 \rho \sigma_{1} \sigma_{2}+\sigma_{2}^{2}} \neq 0$, so this gives $\pi^{2}=-\pi^{1}=0$. Now

$$
E\left[\log Y_{T}^{\pi}\right]=\log Y_{0}+E\left[\int_{0}^{T} Q_{t}\left(\pi_{t}^{1}, \pi_{t}^{2}\right) d t\right] .
$$

Since $\Omega_{\lambda}$ is convex, the maximum of $Q_{t}$ over $\Omega$ is attained at a unique point $\pi_{t}$. Moreover, $\pi_{t}$ depends continuously on $X_{t}$. That $\pi_{t}$ is a piecewise affine function of $X_{t}$ follows from the fact that $\Omega_{\lambda}$ is a polygon.

Proof of Corollary 2.5. Immediate consequence of Theorem 2.3 .

Proof of Corollary 2.6. Immediate consequence of Theorem 2.4 . 


\section{References}

[BO] D. Biagini and B. Øksendal. A general stochastic calculus approach to insider trading. Working Paper, 2003.

[FWY] H. Föllmer, C. T. Wu and M. Yor. Canonical decomposition of linear transformations of two independent Brownian motions motivated by models of insider trading. Stochastic Processes Appl. 84, 137-164, 1999.

[HO] Y. Hu and B. Øksendal. Optimal Smooth Portfolio Selection of An Insider. Working Paper, 2003.

[I] P. Imkeller. Malliavin's calculus in insider models: additional utility and free lunches. Mathematical Finance 13, 153-169, 2003.

[JY] T. Jeulin and M. Yor (editors). Grossissements de filtrations, exemples et applications. Springer Lectures Notes in Math. 1118, Springer Verlag, 1985.

[KP] I. Karatzas and I. Pikovsky. Anticipating portfolio optimization. Adv. Appl. Prob. 28, 1095-1122, 1996.

[KS1] I. Karatzas and S. E. Shreve. Brownian motion and stochastic calculus. Springer-Verlag, New York, 1991.

[KS2] I. Karatzas and S. E. Shreve. Methods of mathematical finance. Springer-Verlag, New York, 1998.

[LNN] J. A. León, R. Navarro and D. Nualart. An anticipating calculus approach to the utility maximization of an insider. Mathematical Finance 13, 171-185, 2003.

[LL] J. Liu and F. A. Longstaff. Losing money on arbitrages: optimal dynamic portfolio choice in markets with arbitrage. Forthcoming, Review of Financial Studies.

[M] R. C. Merton. Continuous-Time Finance. Blackwell, 1992. 